\title{
ESTIMATING THE PROBABILITY OF ERADICATION OF PAINTED APPLE MOTH FROM AUCKLAND
}

\author{
J. M. KEAN ${ }^{1}$ and D. M. SUCKLING ${ }^{2}$ \\ ${ }^{1}$ AgResearch, PO Box 60, Lincoln, New Zealand \\ ${ }^{2}$ HortResearch, PO Box 51, Lincoln, New Zealand \\ Corresponding author: john.kean@agresearch.co.nz
}

\begin{abstract}
A set of assumptions can be used to estimate the confidence that a continuing lack of detection reflects successful eradication of a pest population. This approach is applied to the painted apple moth (Teia anartoides) in Auckland, New Zealand, based on its known population biology and sterile insect recapture results. The analysis suggests that it is extremely unlikely that any wild population might have survived near the core trapping areas beyond mid January 2004, but that there was a significant chance that a small wild population could have remained undetected until then in less intensively trapped areas. The moth trapped in Mt Eden in January 2004 plausibly indicates an undetected population there, but a continued lack of trap catches over the subsequent year suggests that it is very unlikely that any wild populations now remain undetected within the trapping grid. This analysis could be easily adapted for other species targeted for eradication.
\end{abstract}

Keywords: pheromone trapping, Teia anartoides, sterile male technique, dispersal.

\section{INTRODUCTION}

The painted apple moth (PAM), Teia anartoides (Lepidoptera: Lymantriidae), has been the subject of an eradication campaign since its detection in Auckland in 1999. Knowledge of the population biology and dispersal of PAM (e.g. Suckling et al. 2003) can be used to help interpret the continued lack of trap catches and to estimate the probability that the species has been successfully eradicated.

This paper reports an approach whereby a value is calculated for the confidence that a continuing lack of trap catches truly reflects the eradication of the species. A similar analysis was recently reported for tsetse eradication (Barclay \& Hargrove 2005). Such knowledge can inform the incursion response, guide the deployment of monitoring traps, and support any official statement of "successful eradication".

\section{General probability model}

\section{METHODS}

It is firstly assumed that a wild population exists within the trapping grid at its minimum viable population size, denoted $N$. $N$ represents the unstable threshold of the Allee effect (Allee et al. 1949; Liebhold \& Bascompte 2003), with any population declining below $N$ being doomed to extinction, while any population above $N$ tends to increase and so become easier to detect. The most conservative assumption, therefore, is that a wild population persists at constant size $N$ over time.

Given $N$, it is then necessary to estimate the number of trappable individuals that would be present each day, $M_{t}$. In the case of PAM, $M_{t}$ represents the number of adult males. In addition, the capture rate of the trap network, $c_{t}$, must be estimated so that the expected number of individuals caught each day can be calculated as $c_{t} \times M_{t}$. Assuming that 
trapping is random, the probability of catching nothing given that a wild population of size $N$ is present, denoted $p(0 \mid N)$, is given by the zero-term of the Poisson distribution: $p(0 \mid N)=\exp \left(-c_{t} M_{t}\right)$

On the day on which the last male was trapped, it is known that the probability of a wild population being present, $p(N)$, is close to unity. Thereafter, $p(N)$ declines for each day that no males are trapped, by multiplying $p(N)$ from the previous day by $p(0 \mid N)$ for the current day. Consequently, the confidence that eradication has been achieved, $1-p(N)$, increases daily. Successful eradication might be declared once the confidence level exceeds $99 \%$, for example. When another male is caught $p(N)$ is reset to unity.

\section{Applying the model to painted apple moth}

To apply the model on a daily basis, the number of adult males present, $M_{t}$, must be estimated as well as their chance of being trapped, $c_{t}$. The number of adult males produced each day from a population of $N$ immature stages depends on the daily development rate together with the immature and adult survival rates and the sex ratio. Development of PAM males through the egg, larval and pupal stages requires approximately $566^{\circ}$ day above a threshold for development of $9.6^{\circ} \mathrm{C}$ (J. Charles, unpubl. data). Daily thermal accumulation, $T_{b}$, is calculated as a weighted average of the minimum, maximum and mean daily temperatures, $T_{\min }, T_{\max }$, and $T_{\text {mean }}$ :

$$
T_{b}=\left(\left[T_{\min }-b\right]+\left[T_{\max }-b\right]+2\left[T_{\text {mean }}-b\right]\right) / 4
$$

where $b=9.6^{\circ} \mathrm{C}$ is the developmental threshold, and the quantities inside square brackets are set to 0 if negative (Barlow \& Dixon 1980). The daily development rate is then given by the thermal accumulation relative to the total requirement, $q=566^{\circ}$ day. Survival of neonates may be assumed to be 1 by redefining $N$ to represent the population size of successfully maturing individuals. Daily survival of adult males has been measured as approximately $\sigma=20 \%$ in gazebo experiments (Suckling et al. 2003). Given a sex ratio of $\chi=0.5$ males/total, the number of adult males present each day is $M_{t}=\chi N T_{b} / q+\sigma M_{t-1}=N T_{9.6} / 1132+0.2 M_{t-1}$ for PAM.

The daily efficacy of the trap network, $c_{t}$, varies temporally with daily temperatures and spatially with trap density. For PAM, sterile male recapture results provide a benchmark for estimating trapping efficacy. A total of 354,293 sterilised adult male moths were released in Auckland between February 2003 and April 2004. Of these, 3267 were subsequently recovered in traps, suggesting a crude estimate of $c_{t}=0.92 \%$ on average. However, many of these releases were made when weather conditions were not suitable for flight, biasing the estimate downwards. Excluding release dates when none were recaptured, a total of 2168 moths were recaptured from 94755 released, suggesting a recapture rate of $c_{t}=2168 / 94755=2.29 \%$. A more robust estimate of recapture rate can be obtained by examining the sterile male recaptures in relation to daily temperatures. Day degree estimations obtained from equation 2 were weighted according to $20 \%$ daily male survivorship, so that the weighted average was $\left(T_{b}\right.$ (day 1$)+0.2 T_{b}($ day 2$)+0.04 T_{b}$ (day $3)$ )/1.24. The base temperature $b$ was adjusted to maximise the $R^{2}$ correlation with recapture rate, suggesting a base temperature for flight of $17.4^{\circ} \mathrm{C}$ (Fig. 1). Recapture rates were much higher for 2002-03 than for the 2003-04 season, with the slope of the recapture rate function $s=0.0552\left(\mathrm{df}=27, R^{2}=0.781\right)$ for 2002-03, and $s=0.0041(\mathrm{df}=50$, $R^{2}=0.305$ ) for 2003-04. The reason for the difference in recapture rates between the two seasons is not clear, but is probably related to some unmeasured aspect of field conditions, such as rainfall, since there was no change between seasons in the emergence rate or wind tunnel flight fitness of the irradiated males released, nor any decline in the pheromone titre of the females used to bait traps (D.M. Suckling, unpubl. data). The competitiveness of sterile males relative to wild males was estimated as $v=0.66$ from wind tunnel experiments (Suckling et al. 2004). 


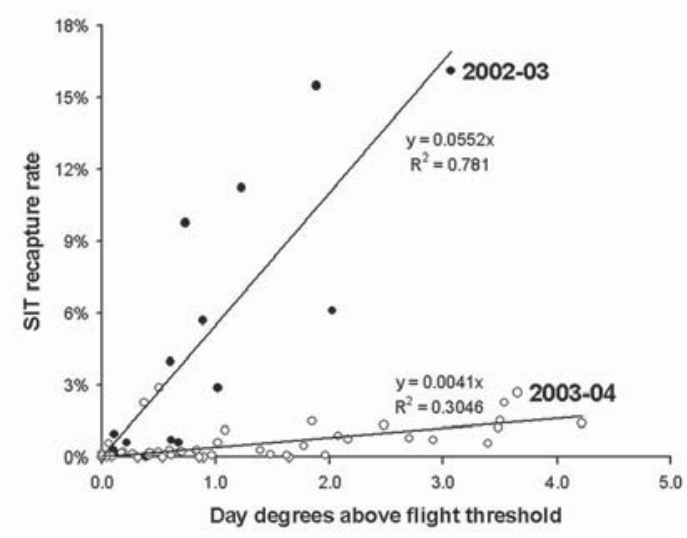

FIGURE 1: Relationship between recapture rate of sterile male releases and weighted temperature on days 1 to 3 after release.

The PAM sterile male releases were all made in areas with suspected wild populations; these also had the highest trap densities, so the sterile male recapture results will tend to overestimate trap efficacy in more sparsely trapped areas. To account for this, the number of recaptures was stratified by distance from the release site, then divided by the number of traps present at that distance, to obtain distance-dependent recapture rates per trap (Fig. 2). Since similar patterns emerged for different Auckland release sites, it was assumed that this relationship is a constant characteristic of the species and environment, being related to species dispersal behaviour. Now, for hypothetical source populations, distance-dependent recapture rates per trap were multiplied by the number of traps present at different distances, and summed to estimate an overall recapture rate. This was then expressed relative to the sterile male release sites to obtain the relative effective trapping density, $d$, for a hypothetical source population. Hence, for a wild population in the core trapping areas (where sterile males were released) $d=1$, while a population in a $500 \mathrm{~m}$ grid of traps has $d=0.37$. Until 2004, the suburb of Mt Eden was sparsely trapped with $d=0.30$, but the trap density there was increased in January 2004 to $d=1.22$. Now the daily probability of males being caught in a delimitation trap is $c_{t}=T_{17.4} \times s \times d / v$.

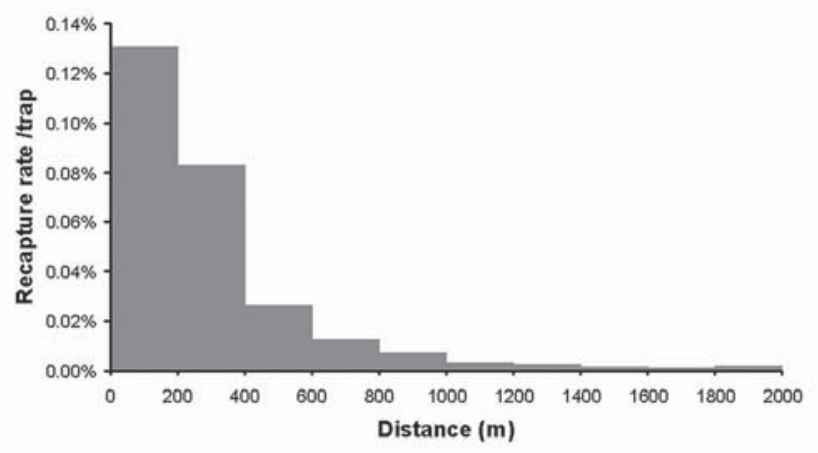

FIGURE 2: Dependence of the proportion of sterile males recaptured per trap on distance from release. 


\section{RESULTS AND DISCUSSION}

Figure 3 shows how the probability of eradication increased after the last wild PAM was caught in 2003. The confidence in eradication increases rapidly in summer, but remains largely constant in winter, because of slow development and limited flight in the cooler temperatures of winter. By mid-January 2004, there was very little chance that a wild population existed within the core areas of Waikumete, Ranui and Hobsonville (Fig. 3, line (a)), but only $79 \%$ confidence that no wild population was present in more sparsely trapped Mt Eden (Fig. 3, line (b)). A male moth was indeed trapped in Mt Eden at that time, resulting in the deployment of additional traps in that area. The extra traps led to a rapid increase in confidence that no further populations exist in Mt Eden (Fig. 3, line (c)), whereas if no additional traps had been deployed then it would have required more than a year of nil trap catches before there could be even $90 \%$ confidence in eradication (Fig. 3 , line (b)). These results assume 2002-03 trap catch rates ( $s=0.0552$, Fig. 1). If 2003-04 trap catch rates are used $(s=0.0041)$ then the results are much more pessimistic. Under this scenario, it will take until early 2006 before there can be reasonable confidence in eradication, even with the new Mt Eden traps (Fig. 3, line (d)).

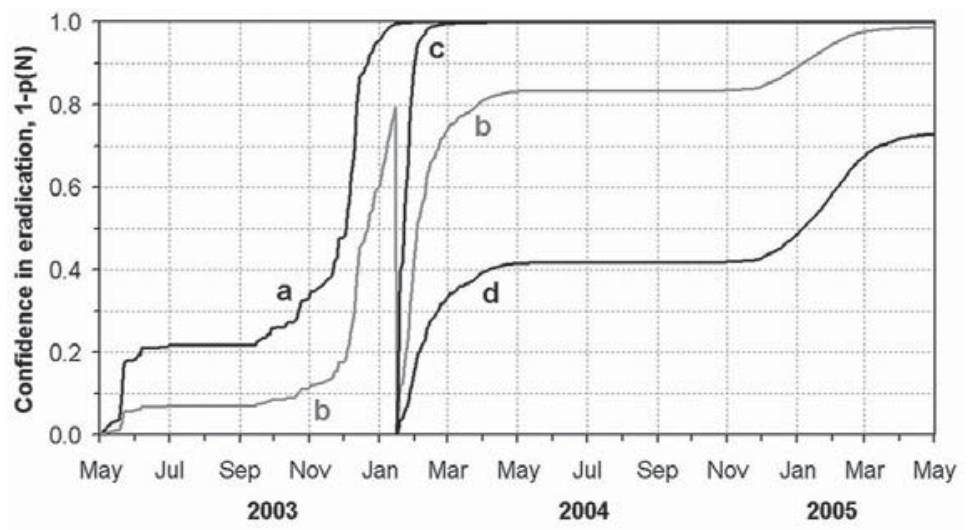

FIGURE 3: Estimated probabilities of painted apple moth eradication given a lack of wild captures. (a) core infestation areas; (b) Mt Eden with 2003 trap layout and 2002-03 recapture rates; (c) Mt Eden with 2005 trap layout and 2002-03 recapture rates; (d) Mt Eden with 2005 trap layout and 2003-04 recapture rates.

It is possible that wild PAM populations remain undetected in more sparsely trapped corners of Auckland, but this seems unlikely. The analysis suggests that where traps are spaced $500 \mathrm{~m}$ apart, each year with no trap catches gives a $98 \%$ decrease in the probability of a population being present, while at $1000 \mathrm{~m}$ spacing there is an annual $46 \%$ decrease in likely presence. Given the rapid increase of historical PAM populations, it is unlikely that wild populations would remain undetected for long periods of time. These results suggest that there can be reasonable confidence that painted apple moth has been successfully eradicated from Auckland, although under current practice eradication is only officially declared two years after the last detection. The methods described may be adapted to aid interpretation of negative trap catches for other species targeted for eradication (e.g. Barclay \& Hargrove 2005). 


\section{ACKNOWLEDGEMENTS}

We thank Quenten Higgan of AgriQuality for providing the trap location data, and numerous HortResearch, Forest Research, and AgriQuality workers for the sterile insect recapture results. Sue Zydenbos and Mike Butcher provided useful comments on the manuscript. This work was supported by the Ministry of Agriculture and Forestry and by the FRST "Improved Biosecurity" programme.

\section{REFERENCES}

Allee WC, Emerson AE, Park O, Park T, Schmidt KP 1949. Principles of animal ecology. Saunders, Philadelphia.

Barclay HJ, Hargrove JW 2005. Probability models to facilitate a declaration of pestfree status, with special reference to tsetse (Diptera: Glossinidae). Bulletin of Entomological Research 95: 1-11.

Barlow ND, Dixon AFG 1980. Simulation of lime aphid population dynamics. Pudoc, Wageningen. $165 \mathrm{pp}$.

Liebhold AM, Bascompte J 2003. The Allee effect, stochastic dynamics and the eradication of alien species. Ecology Letters 6: 133-140.

Suckling DM, Hackett JK, Barrington AM, Chhagen A, Burnip GM, Charles JG, Kay MK, Connolly P, van den Broek LC, Karunaratne L, Pedley RIF, Baird DB 2003. Developing and testing the sterile insect technique against painted apple moth. Unpublished report to MAF Biosecurity NZ. MAF Project Number PAM/601/2002, HortResearch Client Report No. 10877. HortResearch, Palmerston North, New Zealand. $68 \mathrm{p}$.

Suckling DM, Wee S-L, Pedley RIF 2004. Assessing competitive fitness of irradiated painted apple moth Teia anartoides (Lepidoptera: Lymnatriidae). New Zealand Plant Protection 57: 171-176. 